STUDI

FRANCESI

\section{Studi Francesi}

Rivista quadrimestrale fondata da Franco Simone

192 (LXIV | III) | 2020

Texte et images entre Moyen Âge et Renaissance

(manuscrits et imprimés anciens)

\title{
FRANK LESTRINGANT, Bribes d'îles. La littérature en archipel de Benedetto Bordone à Nicolas Bouvier
}

\section{Maurizio Busca}

\section{(2) OpenEdition \\ 1 Journals}

\section{Edizione digitale}

URL: https://journals.openedition.org/studifrancesi/42309

DOI: $10.4000 /$ studifrancesi.42309

ISSN: 2421-5856

\section{Editore}

Rosenberg \& Sellier

\section{Edizione cartacea}

Data di pubblicazione: 1 décembre 2020

Paginazione: 649

ISSN: 0039-2944

\section{Notizia bibliografica digitale}

Maurizio Busca, «FRANK LESTRIngAnt, Bribes d'îles. La littérature en archipel de Benedetto Bordone à Nicolas Bouvier», Studi Francesi [Online], 192 (LXIV | III) | 2020, online dal 01 mars 2021, consultato il 15 octobre 2022. URL: http://journals.openedition.org/studifrancesi/42309; DOI: https://doi.org/10.4000/

studifrancesi.42309

\section{Questo documento è stato generato automaticamente il 15 octobre 2022.}

\section{(c) 9 (1) $\Theta$}

Creative Commons - Attribuzione - Non commerciale - Non opere derivate 4.0 Internazionale - CC BYNC-ND 4.0

https://creativecommons.org/licenses/by-nc-nd/4.0/ 


\title{
FRANK LESTRINGANT, Bribes d'îles. La littérature en archipel de Benedetto Bordone à Nicolas Bouvier
}

\author{
Maurizio Busca
}

\section{NOTIZIA}

FRANK LESTRINGANT, Bribes d'îles. La littérature en archipel de Benedetto Bordone à Nicolas Bouvier, Paris, Classiques Garnier, 2019, «Géographies du monde» 26, 254 pp.

1 A quasi vent'anni dalla pubblicazione del Livre des îles (Atlas et récits insulaires, de la Genèse à Jules Verne, Genève, Droz, 2002), Frank Lestringant ritorna al tema insulare con un volume nel quale convergono ricerche ad ampio raggio, che spaziano dalle opere geografiche e dalla letteratura di viaggio della prima Modernità alla narrativa del secondo Novecento. I «frammenti di isole» eterogenei raccolti nel corso di un esteso lavoro di documentazione vengono ricomposti in «arcipelaghi» testuali e geografici riconducibili a due modelli di organizzazione, centripeto e centrifugo (o «cicladico»e «sporadico»), talvolta concomitanti.

2 La prima delle quattro parti che compongono il volume («En partance pour les îles», pp. 7-29) introduce i materiali su cui saranno condotte le analisi e presenta l'articolazione del saggio, sottolineando la natura peculiare di un oggetto di studio disperso per definizione e annunciando gli approcci ermeneutici che guideranno le indagini. In particolare si rileva il carattere narrativo e poetico dei modelli geografici, e si propone una definizione del genere dell'isolario (cui ampio spazio sarà dedicato nelle pagine successive) quale «récit par les îles» in cui «la fragmentation narrative épouse un ordre cartographique ouvert» (p. 19). La seconda parte («îles fixes», pp. 31-127) affronta da diverse prospettive il tema dell'insularità in un corpus di testi rinascimentali. Nel primo capitolo si ripercorre il dibattito sul problema dell'irregolarità della superficie della sfera terrestre - e quindi dell'esistenza stessa di 
montagne e isole - negli scritti di Coronelli, Porcacchi, Merula e Münster. Il secondo capitolo introduce alle rappresentazioni delle isole in opere geografiche e resoconti di viaggio (in particolare Thevet e Pigafetta), mentre il terzo prende in esame il fenomeno diffuso, presso moralisti e narratori (De Lancre, Marguerite de Navarre, Belleforest...), dell'associazione delle isole ad un immaginario demoniaco. Il quarto e il quinto capitolo sono dedicati rispettivamente all'Isolario di Leandro Alberti e al suo reimpiego da parte di Thevet, e alla prima «robinsonnade» della letteratura francese: la storia, narrata da Marguerite de Navarre, Belleforest e Thevet, della giovane esiliata su un'isola deserta canadese nel corso della spedizione di Roberval del 1542. Nella terza e quarta parte («Îles instables», pp. 129-176; «Bribes d'îles», pp. 177-221) le analisi si estendono fino alla seconda metà del secolo scorso percorrendo opere di Gédéon Flournois, Voltaire, Morelly, Defoe, Wyss, Hugo, Giraudoux, Saint-Exupéry, Michaux, Prévert, Calvino, Bouvier e Glissant. In queste pagine le ricerche procedono in maniera ovviamente non sistematica, ma la puntuale messa in luce di fenomeni di continuità e trasformazione dell'immaginario insulare nella letteratura moderna e contemporanea risulta certamente convincente. 